\title{
B spectroscopy with NRQCD and HISQ
}

\author{
Eric B. Gregory ${ }^{* \dagger}$ \\ University of Glasgow \\ E-mail: egregory@ucy.ac.cy
}

\section{Christine.T.H. Davies}

University of Glasgow

\section{Eduardo. Follana}

Universidad de Zaragoza

\section{Elvira. Gámiz}

University of Illinois

\section{lain Kendall}

University of Glasgow

\section{G. Peter Lepage}

Cornell University

\section{Heechang $\mathrm{Na}$}

The Ohio State University

\section{Junko Shigemitsu}

The Ohio State University

\section{Kit Y. Wong}

University of Glasgow

Using NRQCD $b$ quarks and HISQ light, strange and charm quarks we have calculated $B$ meson masses and $B^{*}-B$ splittings. We quote results for a range of lattice spacings and sea quark masses, enabling controlled extrapolation to the physical point. Since the $b$ quark masses and lattice spacing are fixed from the $\Upsilon$ and other meson masses, this allows accurate, parameter-free tests of $B, B_{s}$, and $B_{c}$ masses against experiment. We can also predict the mass of the $B_{c}^{*}$ meson

The XXVII International Symposium on Lattice Field Theory - LAT2009

July 26-31 2009

Peking University, Beijing, China

\footnotetext{
* Speaker.

${ }^{\dagger}$ Current address: Department of Physics, University of Cyprus, P.O. Box 20357, 1678 Nicosia, CYPRUS
} 


\begin{tabular}{llllllll}
\hline \hline Set & $\beta$ & $r_{1} / a$ & $a u_{0} m_{0 l}^{a s q}$ & $a u_{0} m_{0 s}^{a s q}$ & $L / a$ & $T / a$ & $N_{\text {conf }} \times N_{t}$ \\
\hline 1 & 6.572 & $2.152(5)$ & 0.0097 & 0.0484 & 16 & 48 & $624 \times 2$ \\
2 & 6.586 & $2.138(4)$ & 0.0194 & 0.0484 & 16 & 48 & $628 \times 2$ \\
\hline 3 & 6.760 & $2.647(3)$ & 0.005 & 0.05 & 24 & 64 & $507 \times 2$ \\
4 & 6.760 & $2.618(3)$ & 0.01 & 0.05 & 20 & 64 & $589 \times 2$ \\
\hline 5 & 7.090 & $3.699(3)$ & 0.0062 & 0.031 & 28 & 96 & $530 \times 4$ \\
\hline \hline
\end{tabular}

Table 1: Ensembles (sets) of MILC configurations used with gauge coupling $\beta$, size $L^{3} \times T$ and sea masses ( $\times$ tadpole parameter, $u_{0}$ ) $m_{0 l}^{a s q}$ and $m_{0 s}^{a s q}$. Column 3 is the lattice spacing values in units of $r_{1}$ after "smoothing' [4]. Column 8 gives the number of configurations and time sources per configuration that we used for calculating correlators. On set 5 only half the number were used for light quarks.

\section{Introduction}

The $B$ meson sector is a compelling target for lattice calculations for a variety of reasons. There are a variety of so-called "gold-plated" states — those states which are narrow and hadronically stable, as well as being experimentally accessible. In calculating these properties of states on the lattice there are no free parameters; $m_{\pi}, m_{K}, m_{\eta_{c}}$ and $m_{\Upsilon}$ calibrate the masses of the light, strange, charm and bottom quarks respectively, and $\Upsilon$ splittings and other meson masses calibrate the lattice spacing[1, 2, 3].

Precision $B$ meson spectroscopy is a key ingredient in precision calculation of decay constants and form factors, ingredients in CKM matrix element determination and testing of the standard model.

\section{Simulation Methods}

We use five different ensembles of gauge configurations with $2+1$ flavors of dynamical ASQTAD sea quarks, generated by the MILC collaboration. The ensembles, listed in Table 1, represent three lattice spacings, labeled very-coarse, coarse, and fine.

On each configuration we generate and store random-wall HISQ propagators for several source time slices for light, strange and charm quarks:

$$
g^{\mathrm{HISQ}}\left(\mathbf{x}, t_{0}\right)=M_{x, x^{\prime}}^{-1} \eta\left(t_{0}\right)_{x^{\prime}}
$$

where $\eta\left(t_{0}\right)_{x^{\prime}}$ is a three-component complex unit vector of random numbers at each site of the source timeslice, $t_{0}$ and zero elsewhere.

The HISQ action uses an additional application of the fattening step of the ASQTAD formulation, reducing discretization errors to the extent that it is possible to simulate relativistic charm quarks on configurations of modest lattice spacing.

Bottom quarks are too massive to simulate relativistically on these lattices. However within bound states, the $b$ quark is generally slow enough $\left(v_{b} / c \sim 0.01\right.$ in $\left.B_{c}\right)$ to treat non-relativistically. The use of the NRQCD action for b quarks is a well-developed procedure. [5, 6, 7] 
We evolve the NRQCD propagator recursively:

$$
G_{i}(x, t+1)=\left(1-\frac{\delta H}{2}\right)\left(1-\frac{H_{0}}{2 n}\right)^{n} U_{t}^{\dagger}(x)\left(1-\frac{H_{0}}{2 n}\right)^{n}\left(1-\frac{\delta H}{2}\right) G_{i}(x, t),
$$

with

$$
\begin{aligned}
\delta H= & -c_{1} \frac{\left(\Delta^{(2)}\right)^{2}}{8\left(M^{0}\right)^{3}}+c_{2} \frac{i g}{8\left(M^{0}\right)^{3}}(\tilde{\Delta} \cdot \tilde{E}-\tilde{E} \cdot \tilde{\Delta})-c_{3} \frac{i g}{8\left(M^{0}\right)^{3}} \sigma \cdot(\tilde{\Delta} \times \tilde{E}-\tilde{E} \times \tilde{\Delta}) \\
& -c_{4} \frac{g}{2 M^{0}} \sigma \cdot \tilde{\mathbf{B}}+c_{5} \frac{a^{2} \Delta^{(4)}}{24 M^{0}}-c_{6} \frac{a\left(\Delta^{(2)}\right)^{2}}{16 n\left(M^{0}\right)^{2}} .
\end{aligned}
$$

The tilde expressions $\tilde{\mathbf{E}}$ and $\tilde{\mathbf{B}}$ are improved versions of the naive lattice chromo-electric and chromo-magnetic fields, $\mathbf{E}$ and $\mathbf{B}$. We use the tree-level values of $c_{i}=1$ for the constants.

To double statistics, we evolve the NRQCD propagator both forward and backward across the lattice from the source timeslice.

As we have used a random-wall source for the HISQ propagators, it is critical that we initialize the NRQCD $b$ propagators with the same random-wall function $\eta\left(t_{0}\right)_{x^{\prime}}$ as we used for the HISQ propagators. This is slightly non-trivial in that the HISQ staggered fermions, and the random wall vector $\eta\left(t_{0}\right)_{x^{\prime}}$, have one Dirac component per site, while the NRQCD $b$ quarks have two upper and/or two lower Dirac components. The trick is to undo the staggering transformation by multiplying the noise source $\eta\left(t_{0}\right)_{x^{\prime}}$ at each site with the four-component staggering operator:

$$
\Omega(x)=\gamma_{0}^{x_{0}} \gamma_{1}^{x_{1}} \gamma_{2}^{x_{2}} \gamma_{3}^{x_{3}}
$$

Furthermore, to isolate the meson ground-state, we smear the $b$ propagator source with a Gaussian smearing function of varying radii $r_{i}$. Therefore, on timeslice $t_{0}$ we initialize the NRQCD propagator as:

$$
G_{i}^{\mathrm{NRQCD}}\left(\mathbf{x}, t_{0}\right)=\sum_{x^{\prime}} S\left(\left|x-x^{\prime}\right| ; r_{i}\right) \eta_{x^{\prime}}\left(t_{0}\right) \Omega\left(x^{\prime}\right) \Gamma,
$$

where $\Gamma$ is an element of the Dirac algebra chosen to project out a desired meson state.

At the sink end we must also multiply $\Omega(x)$ back into the HISQ propagator so that we can get a multi-Dirac-component object to trace with the NRQCD $b$ propagator:

$$
G^{\mathrm{HISQ}}(\mathbf{x}, t)_{a b}=g^{\mathrm{HISQ}}\left(\mathbf{x}, t_{0}\right)_{x} \Omega(\mathbf{x}, t)_{a b}
$$

Then our $B$ meson correlator matrix is:

$$
C_{\Gamma}\left(t-t_{0}\right)_{i j}=\sum_{\mathbf{x}} G^{\mathrm{HISQ}^{\dagger}}(\mathbf{x}, t) \Gamma S\left(\left|x-x^{\prime}\right| ; r_{j}\right) G_{i}^{\mathrm{NRQCD}}\left(\mathbf{x}^{\prime}, t\right) .
$$

\section{Analysis}

We extract $B$ meson energies from the matrix of correlators (2.77) using a Bayesian factorizing fit to the form

$$
C_{\Gamma}\left(t-t_{0}\right)_{i j}=\sum_{k=1}^{N_{\exp }} a_{i, k} a_{j, k}^{*} e^{-E_{k}\left(t-t_{0}\right)}+\sum_{k^{\prime}=1}^{N_{\exp }-1} b_{i, k^{\prime}} b_{j, k^{\prime}}^{*}(-1)^{\left(t-t_{0}\right)} e^{-E_{k^{\prime}}^{\prime}\left(t-t_{0}\right)}
$$


where the second term fits the oscillating component inherent in staggered meson correlators.

We look for high-confidence fits stable with respect to varying $N_{\text {exp }}$, and $t_{\min }$ of the fit range. Where possible we simultaneously fit all the correlators coming from the same ensemble, to better account for correlated errors.

In practice we fit $B$ (light) and $B_{s}$ together in all cases except the fine ensemble (set 5). We fit a $3 \times 3$ matrix of smeared correlators in all cases except for the $B_{c}$ fits on the very-coarse ensembles (sets 1 and 2). We always fit the pseudoscalar and vector states simultaneously.

We are interested in the ground-state energies $E_{0}$ and the ground-state of the oscillating paritypartner channel $E_{0}^{\prime}$. A factor of $\gamma_{0} \gamma_{5}$ relates the spin structure $\Gamma$ of the direct channel with that of the parity partner channel, $\Gamma^{\prime}$. In this way a measured pseudoscalar correlator also contains a scalar meson correlator, and a vector correlator also contains an axial vector correlator at no extra cost.

Because the relativistic relation between energy and mass does not hold for NRQCD $b$ quarks, there is an unknown energy shift between the physical masses we are interested in and the the fitted energies. Instead we measure the splitting between the state of interest and a similar state with the same NRQCD quark content.

We convert this splitting to physical units using $r_{1}=0.3133(23) \mathrm{fm}$ [1], giving a $0.7 \%$ uncertainty in any measured splitting in our lattice calculation. Hence we can minimize the scale-setting error by choosing comparison states as close as possible to the state of interest. We are perfectly free to construct a fictitious comparison state which is a composite of real states, provided all components have well-known experimental and lattice measurement for calibration.

We consider three methods to determine the $B_{s}$ and $B_{c}$ masses:

$$
\begin{gathered}
M_{B_{s / c}}=\left(E_{B_{s} / c}-\frac{1}{2} E_{b \bar{b}}\right)_{\text {latt }}+\frac{1}{2} M_{b \bar{b}} \\
M_{B_{c}}=\left(E_{B_{c}}-\frac{1}{2}\left(E_{b \bar{b}}+E_{c \bar{c}}\right)\right)_{\text {latt }}+\frac{1}{2}\left(M_{b \bar{b}}+M_{c \bar{c}}\right) \\
M_{B_{c}}=\left(E_{B_{c}}-\left(E_{B_{s}}+E_{D_{s}}-E_{\eta_{s}}\right)\right)_{\text {latt }}+\left(M_{B_{s}}+M_{D_{s}}-M_{\eta_{s}}\right)
\end{gathered}
$$

Here $E_{b \bar{b}}$, for example, refers to the spin-averaged lattice energy of $b \bar{b}$ states. In each equation we must apply the lattice scale $a^{-1}$ (and its uncertainty) to the expression in the () latt only.

Where the subtraction compares states with different electromagnetic charge structures we must estimate the adjustment necessary to account for electromagnetic effects.

\section{Results and discussion}

\subsection{Pseudoscalar states}

In practice Method Ii is the only one applicable to $B_{s}$ spectroscopy. We extract the lattice energies of the $B_{s}$ states from each of the ensembles, convert to physical masses via expression $[$.

After the recent, more precise determination of $r_{1}$ [1], it has become apparent that both the $s$ quark mass and the $b$ quark mass were tuned too high. Method I for $M_{B_{s}}$ is particularly sensitive to the mistuned quarks. We have estimated the effect of the mistuned quark masses by substituting into Method 1 mesons with different valence masses. We estimate that for the very coarse, coarse 

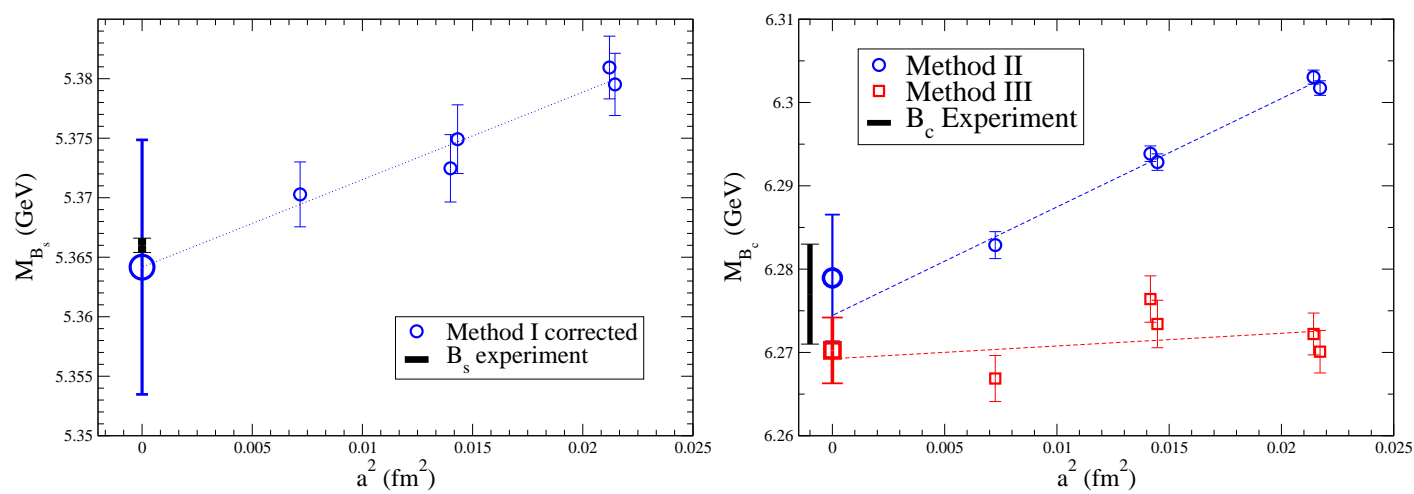

Figure 1: Lattice calculations for $M_{B_{s}}$ (left) $M_{B_{c}}$ (right) and with energy shift subtracted and resulting masses extrapolated in $a^{2}$ to the physical point. For $M_{B_{s}}$ we correct the finite- $a^{2}$ points for $b$ and $s$ mass mistuning before extrapolation. For $M_{B_{c}}$ the continuum points are corrected upwards for electromagnetic effects by $4.5 \mathrm{MeV}$ and $1 \mathrm{MeV}$ for Methods I and II respectively. Error bars on extrapolated points reflect total errors.

and fine ensembles, the too-large strange mass pushes up $M_{B_{s}}$ by $7.5,10$ and $9 \mathrm{MeV}$, respectively. The $b$ mistunings bias $M_{B_{s}}$ up by $10.5,13$, and $15 \mathrm{MeV}$ on the same ensembles. We correct for these biases in the finite $a$ calculations, and then extrapolate, estimating an additional systematic uncertainty of $10 \mathrm{MeV}$ on the extrapolated value, giving:

$$
M_{B_{s}}=5.341(4)(10) \mathrm{GeV},
$$

with the first error being statistical and the second, dominant, error being the quark-tuning systematic error. Figure 1 (left) illustrates the extrapolation.

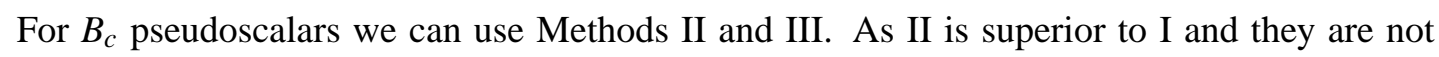
linearly independent we do not also consider 1 here. We again extrapolate in $a^{2}$ to the continuum for each.

We correct for the electromagnetic structure mismatch. Method [1] compares neutral $b \bar{b}$ and $c \bar{c}$ states with the charged $B_{c}$ state. We calculate that this mismatch causes an underestimate of $M_{B_{c}}$ by $\sim 4.5 \pm 2 \mathrm{MeV}$. In Method $\amalg$, comparing similarly charged $B_{c}$ and $D_{s}$ introduces an underestimate of $\sim 1 \pm 1 \mathrm{MeV}$.

After correcting the electromagnetic contribution we get:

$$
\begin{aligned}
M_{B_{c}}(I I) & =6.279(2)(1)(5)(2) \mathrm{GeV} \\
M_{B_{c}}(I I I) & =6.268(4)(6)(1)(1) \mathrm{GeV},
\end{aligned}
$$

where the errors are (statistical)( $\left.r_{1}\right)(\mathrm{NRQCD})(\mathrm{EM})$. The agreement between the two independent subtraction methods is a strong test of our control of systematics. Because the ()$_{\text {latt }}$ term is very small, both are quite insensitive to $b$ and $s$ tuning, and no further subtraction is necessary. Results from both methods are in excellent agreement with the PDG average of 6.277(6) GeV[8]. See Figure 1, right.

The HISQ $c$ quark seems to be the source of the strong discretization effects in Method II, which go as $\alpha_{s}(v / c)^{2}\left(a m_{c}\right)^{2}$. The $c$ quark is more relativistic inside the $B_{c}$ than in a $c \bar{c}$, so these errors do not cancel exactly, but should vanish in the continuum. 


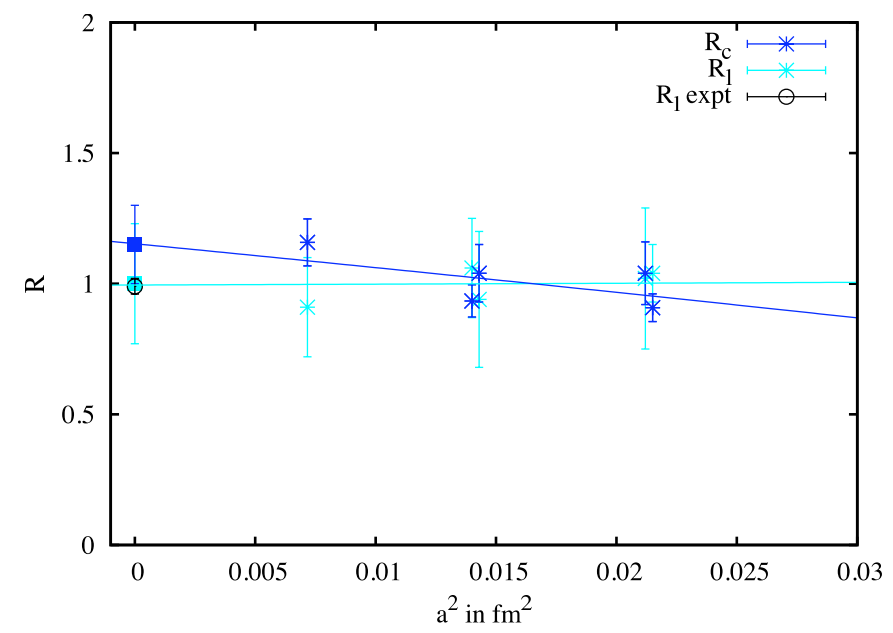

Figure 2: The extrapolation of the ratio $R$ to the continuum for both $B_{c}^{*}$ and $B^{*}$.

\subsection{Vector states}

For the $B^{*}$ states there is an obvious method of correcting for the NRQCD energy shift compare to the nearby $B$ pseudoscalar states to get the hyperfine splitting. The remaining complication is that since the $\sigma \cdot B$ term in the NRQCD action generates the $B^{*}-B$ splittings, radiative corrections to this term could generate a multiplicative correction to the splitting. (Recall we have used the tree-level $c_{4}$.) We therefore use the hyperfine splitting of the $B_{s}$ system as calibration for that of the $B_{c}$ system and calculate:

$$
R_{c}=\frac{E_{B_{c}^{*}}-E_{B_{c}}}{E_{B_{s}^{*}}-E_{B_{s}}}
$$

which will cancel all of the NRQCD energy shifts, multiplicative corrections, and scale-setting error. We extrapolate to $a^{2}=0$, multiply by the PDG average value of $M_{B_{s}^{*}}-M_{B_{s}}=46.1(1.5) \mathrm{MeV}$ [8], and add the experimental $B_{c}$ mass, giving us a prediction of the $B_{c}^{*}$ mass of $M_{B_{c}^{*}}=6.330(7)(2)(6)$ $\mathrm{GeV}$. As a check we also calculate $R_{l}$ with light quark $B$ and $B^{*}$ states. A complete discussion of the $B_{c}$ hyperfine splitting calculation can be found in [9].

\subsection{Scalar states}

As mentioned in Section 3, the oscillating component of the pseudoscalar correlators gives us the scalar states.

We extract the $E_{0^{+}}-E_{0^{-}}$splittings directly in the simultaneous fits. Converting to physical units we again extrapolate in $a^{2}$ to the continuum and find:

$$
\begin{aligned}
& \Delta M_{B_{s}}\left(0^{+}-0^{-}\right)=0.41(2) \mathrm{GeV} \\
& \Delta M_{B_{c}}\left(0^{+}-0^{-}\right)=0.44(7) \mathrm{GeV},
\end{aligned}
$$

quoting statistical errors only. As this splitting is generated by the kinetic term, it should acquire no multiplicative renormalization, and most systematics should cancel. The $O^{+} B_{c}$ state lies about $400 \mathrm{MeV}$ below the $B+D$ threshold so it should be a narrow state. It is less clear whether the $0^{+} B_{s}$ state is below the $B+K$ state, but in any case it should be close enough to the $B+K$ that it should also be a narrow state. See Figure 3 , right and left, respectively. 

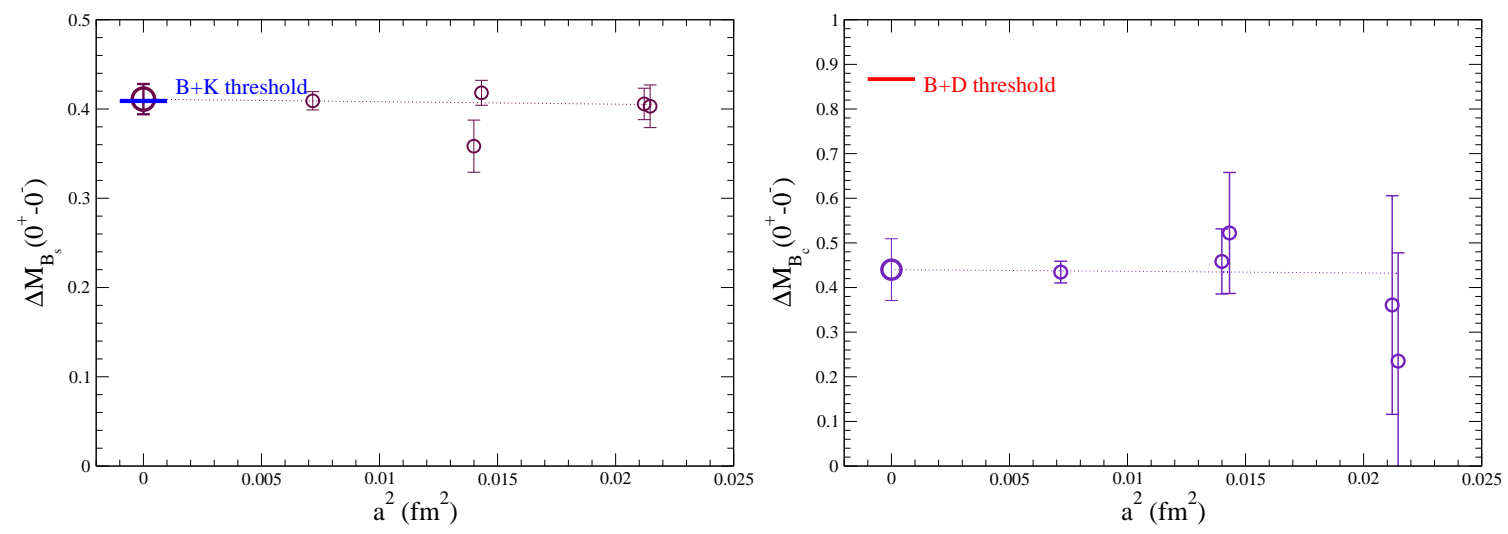

Figure 3: The splitting between the scalar and pseudoscalar states for $B_{S}$ (left) and $B_{c}$ (right). Values are extrapolated to the continuum. Shown on each are the relevant hadronic thresholds.

\section{Conclusions}

We have shown preliminary results of precise lattice calculations of pseudoscalar masses in the $B_{s}$ and $B_{c}$ system, and of vector-pseudoscalar and scalar-pseudoscalar splittings. Our calculation of $M_{B_{s}}$ and $M_{B_{c}}$ agree within errors with experimental measurements of these states. Some work remains to fully understand the systematic errors and biases related to mistuning of quark masses.

Our calculations of the $B_{s}$ and $B_{c}$ scalars and the $B_{c}^{*}$ vector constitute predictions of the masses of these states before experimental measurement.

The precision and accuracy of these results reaffirms that the combination of HISQ light quarks and NRQCD $b$ quarks is a powerful lattice technique. Further work will complete the exploration of the lowest $B, B_{s}$ and $B_{c}$ states, and then apply these techniques to the calculation of form-factors and decay constants relevant to weak-matrix elements.

\section{References}

[1] C. T. H. Davies, E. Follana, I. D. Kendall, G. P. Lepage and C. McNeile, [arXiv:0910.1229 [hep-lat]].

[2] E. Follana et al. Phys. Rev. D 75, 054502 (2007) [arXiv:hep-lat/0610092].

[3] E. Follana, C. T. H. Davies, G. P. Lepage and J. Shigemitsu Phys. Rev. Lett. 100, 062002 (2008) [arXiv:0706.1726 [hep-lat]].

[4] A. Bazavov et al, arXiv:0903.3598.

[5] B. A. Thacker and G. P. Lepage, Phys. Rev. D 43, 196 (1991).

[6] G. P. Lepage et al, Phys. Rev. D 46, 4052 (1992) [arXiv:hep-lat/9205007].

[7] C. T. H. Davies et al, Phys. Rev. D50:6963 (1994) [arXiv:hep-lat/9406017].

[8] Particle Data Group, http://pdg.lbl.gov.

[9] E. B. Gregory et al., arXiv:0909.4462 [hep-lat]. 